\title{
A novel method to isolate mesenchymal stem cells from bone marrow in a closed system using a device made by nonwoven fabric.
}

\section{$\operatorname{AUTHOR(S):~}$}

Ito, Kinya; Aoyama, Tomoki; Fukiage, Kenichi; Otsuka, Seiji; Furu, Moritoshi; Jin, Yonghui; Nasu, Akira; ... Otsuka, Takanobu; Nakamura, Takashi; Toguchida, Junya

\section{CITATION:}

Ito, Kinya ...[et al]. A novel method to isolate mesenchymal stem cells from bone marrow in a closed system using a device made by nonwoven fabric.. Tissue engineering. Part C, Methods 2010, 16(1): 81-91

\section{ISSUE DATE:}

2010-02

URL:

http://hdl.handle.net/2433/159718

\section{RIGHT:}

This is a copy of an article published in the Tissue Engineering Part C.; (C) 2010 Mary Ann Liebert, Inc; "Tissue Engineering Part C" is available online at: http://www.liebertonline.com. 


\title{
A Novel Method to Isolate Mesenchymal Stem Cells from Bone Marrow in a Closed System Using a Device Made by Nonwoven Fabric
}

\author{
Kinya Ito, M.D.,, ${ }^{1,2}$ Tomoki Aoyama, M.D., Ph.D., ${ }^{1}$ Kenichi Fukiage, M.D., ${ }^{1,3}$ Seiji Otsuka, M.D., Ph.D.,, ${ }^{1,2}$ \\ Moritoshi Furu, M.D., ${ }^{1,3}$ Yonghui Jin, M.D., Akira Nasu, M.D., ${ }^{1,3}$ Michiko Ueda, ${ }^{1}$ Yasunari Kasai, ${ }^{4}$ \\ Eishi Ashihara, M.D., Ph.D., ${ }^{4}$ Shinya Kimura, M.D., Ph.D., ${ }^{4}$ Taira Maekawa, M.D., Ph.D., ${ }^{4}$ \\ Akira Kobayashi, Ph.D., 5 Shinya Yoshida, Ph.D., ${ }^{5}$ Hideo Niwa, Ph.D., ${ }^{5}$ Takanobu Otsuka, M.D., Ph.D., ${ }^{2}$ \\ Takashi Nakamura, M.D., Ph.D., ${ }^{3}$ and Junya Toguchida, M.D., Ph.D.
}

Bone marrow stromal cells (BMSCs) include cells with multidirectional differentiation potential described as mesenchymal stem cells. For clinical use, it is important to develop a way to isolate BMSCs from bone marrow in a closed system without centrifugation. After screening 200 biomaterials, we developed a device containing a nonwoven fabric filter composed of rayon and polyethylene. The filter selectively traps BMSCs among mononuclear cells in bone marrow based on affinity, not cell size. The cells are then recovered by the retrograde flow. Using canine and human bone marrow cells, the biological properties of BMSCs isolated by the device were compared with those obtained by conventional methods using centrifugation. The total number isolated by the device was larger, as was the number of $\mathrm{CD} 106^{+} / \mathrm{STRO}-1^{+}$double-positive cells. The cells showed osteogenic, chondrogenic, and adipogenic differentiation potential in vitro. Finally, the direct transplantation of cells isolated by the device without in vitro cultivation accelerated bone regeneration in a canine model of osteonecrosis in vivo. The proposed method is rapid and efficient, does not require a biological clean area, and will be useful for the clinical application of mesenchymal stem cells in bone marrow.

\section{Introduction}

B ONe marrow stromal Cells (BMSCs) include cells with multidirectional differentiation potential such as mesenchymal stem cells (MSCs), multipotent adult progenitor cells, and marrow-isolated adult multilineage inducible cells, although it is not yet clear whether these cell types are distinct or overlap. ${ }^{1,2}$ In spite of such ambiguity, BMSCderived multipotent cells have been used in various fields of regenerative medicine. For clinical applications, however, it is critical to develop a method separating BMSCs containing multipotent cells from other types of cells in bone marrow that is simple, safe, inexpensive, and achievable in a closed system. The most popular way to isolate BMSCs is the density gradient method using a detergent such as sucrose ${ }^{3}$ although the process is technically demanding and time consuming. Simple centrifugation with low gravity can also separate mononuclear cells (MNCs) from erythrocytes, and BMSCs can be selected through adherence to plastic dishes. ${ }^{4}$
This method, however, still needs centrifugation, which makes it difficult to perform in a closed system, and therefore requires a biologically clean room.

To avoid the need for centrifugation, we have attempted to develop a device that can isolate BMSCs by filtration, and have selected nonwoven fabrics as biomaterials. Nonwoven fabrics are engineered materials that provide specific functions such as absorbency, liquid repellency, resilience, stretch, softness, strength, flame retardancy, washability, and cushioning. ${ }^{5}$ As for clinical applications, nonwoven fabrics are used mainly for two purposes, as scaffolds and filters. A number of studies have used nonwoven fabrics as scaffolds for tissue regeneration. ${ }^{5-7}$ The material and diameter of the fabric are important for cell attachment. 6,8 For example, polyethylene terephthalate with fibers $9.0 \mu \mathrm{m}$ in diameter is ideal for the osteogenic differentiation of MSCs. ${ }^{6}$ As filters, nonwoven fabrics have been used to sterilize medical materials. ${ }^{9}$ Lymphocytapheresis is a form of treatment for patients with autoimmune diseases, in which lymphocytes in

\footnotetext{
${ }^{1}$ Institute for Frontier Medical Sciences, Kyoto University, Kyoto, Japan.

${ }^{2}$ Department of Orthopaedic Surgery, Graduate School of Medical Sciences, Nagoya City University, Nagoya, Japan.

${ }^{3}$ Department of Orthopaedic Surgery, Graduate School of Medicine, Kyoto University, Kyoto, Japan.

${ }^{4}$ Department of Transfusion Medicine and Cell Therapy, Kyoto University Hospital, Kyoto, Japan.

${ }^{5}$ Kaneka Co., Osaka, Japan.
} 
peripheral blood are removed to reduce immunoreactions. To replace the centrifugation process in lymphocytapheresis, a filtration method using nonwoven fabrics has been developed. ${ }^{10}$ The trapping effect is attributed to the affinity of the material for cells and the size of the material's fibers, with almost all leukocytes trapped when the fibers are less than $3 \mu \mathrm{m}$ in diameter. ${ }^{10,11}$

As pure mechanical trapping based on cell size can damage cell membranes, we have tried to develop a filter that traps MSCs through affinity and here show that a new device composed of rayon-polyethylene nonwoven fabrics can isolate BMSCs from bone marrow aspirates, which contain cells compatible with multipotent cells in vitro and in vivo.

\section{Materials and Methods}

\section{Screening of materials}

The basic concept behind our approach is to trap BMSCs among bone marrow aspirates on a filter, and collect them using a retrograde flow (Fig. 1A). Biomaterials for the filter were screened based on microscopic structure (evenness), diameter (over $10 \mu \mathrm{m}$ ), weight (over $50 \mathrm{~g} / \mathrm{m}^{2}$ ), bio-safety, and availability (as the first screening). Potentially suitable biomaterials were further screened by conducting colony-forming unit (CFU) assays described later using swine bone marrow cells in three steps (as the second, third, and fourth screening; Supplemental Fig. S1, available online at www.liebertonline.com).

\section{Electron microscopic analyses}

After bone marrow had permeated through it, the filter was treated with $2.5 \mathrm{wt} \%$ glutaraldehyde in saline for 2 days at $4{ }^{\circ} \mathrm{C}$. The samples were dried by passing them through a series of graded-alcohol-saline solutions. Finally, the solution was changed to $100 \%$ t-butanol, and the samples were freeze-dried for $10 \mathrm{~h}$ at $-5^{\circ} \mathrm{C}$. The dried membranes were coated with gold-palladium and examined using a S-3000N scanning electron microscope (Hitachi, Tokyo, Japan).

\section{Harvesting of bone marrow}

Humans. Ten milliliters of bone marrow was taken from iliac crests of donors who received orthopedic operative procedures requiring autologous bone grafts from iliac crests. The donors had no history of concurrent illness or of medication that could affect bone metabolism. The Ethics Committee of the Faculty of Medicine, Kyoto University, approved the procedure, and informed consent was obtained from each donor according to the declaration of Helsinki.

Dogs. Ten milliliters of bone marrow was aspirated from iliac crests of male beagle dogs $(10-13 \mathrm{~kg})$ under intravenous anesthesia. The experiments with animals were approved by the institutional animal research committee, and performed according to the Guidelines for Animal Experiments of Kyoto University.

\section{Preparation of MNCs}

Human marrow samples were divided equally into three aliquots ( $3 \mathrm{~mL}$ each). One aliquot was applied to the device (method D), which had been saturated with saline. After washing the filter with $10 \mathrm{~mL}$ of saline, trapped cells were collected by a retrograde flow of the culture medium into a collection bag (Fig. 1A). The second aliquot was suspended in $5 \mathrm{~mL}$ of $\alpha$-minimum essential medium GlutaMAX (Invitrogen, Carlsbad CA, ) with 10\% fetal bovine serum (Hyclone, South Logan, UT). The suspension was centrifuged at $1200 \mathrm{rpm}$ for $5 \mathrm{~min}$. The supernatant and the buffy coat were re-suspended in another tube with $5 \mathrm{~mL}$ of $\alpha$ minimum essential medium GlutaMAX with $10 \%$ fetal bovine serum. This method is hereafter designated method $S$ (simple centrifugation). ${ }^{4,12}$ The final aliquot of bone marrow was fractionated by centrifugation over a density cushion using a Ficoll gradient (GE Healthcare Life Sciences, Piscataway, NJ), as described. ${ }^{3}$ The interface layer was harvested and washed twice with $10 \mathrm{~mL}$ of Hank's buffer solution. This method is hereafter designated method F (Ficoll gradient).

\section{Collection efficacy}

Cells trapped on the filter and collected by retrograde flow in the collection bag were designated the collected fraction, and cells flowing through the filter into the flow-through bag were designated the flow-through fraction (Fig. 1A). Collection efficacy was determined as the ratio of the number of cells in each fraction to that in the applied bone marrow.

\section{CFU assay}

For the CFU assay, the MNCs were diluted and plated in 60$\mathrm{mm}$ cell culture dishes at a density of $2.5 \times 10^{5}$ cells $/$ dish and incubated for 14 days $(n=3)$. The cells were fixed with methanol and stained with a $0.05 \%$ crystal violet solution. We counted the number of colony with a diameter more than $4 \mathrm{~mm}$.

\section{Flow cytometry}

Cells were stained at room temperature for $30 \mathrm{~min}$ with the following antibodies: CD10-phycoerythrin (PE), CD34allophycocyanin (APC), CD45-peridinin chlorophyll protein (PerCP-Cy5.5), CD106-fluorescein isothiocyanate (FITC), CD166-PE (BD Biosciences, San Jose, CA), CD90-FITC (Beckman Coulter, Fullerton, CA), CD271-APC (Miltenyi Biotec, Bergisch Gladbach, Germany), and STRO-1-PE (Santa Cruz Biotechnology, Santa Cruz, CA). They were subsequently subjected to flow cytometry using a FACS Calibur instrument (BD Biosciences).

\section{Induction of differentiation and histochemical evaluating}

Differentiation was induced using the standard method. ${ }^{13}$ The differentiation potential was evaluated as below.

Osteogenic differentiation: Calcified nodules were evaluated by alizarin red staining, and calcium content was quantified.

Adipogenic differentiation: Intracellular lipid droplets were stained with oil red-O, and the amount of triglyceride was quantified.

Chondrogenic differentiation: Cartilage matrix was evaluated by alcian blue staining, and the amount of glycosaminoglycan was quantified.

These analyses were described previously. ${ }^{14}$

\section{Reverse transcription-polymerase chain reaction}

Total RNA was extracted using RNeasy Kit (Qiagen, Hilden, Germany). All reverse transcription reactions were 


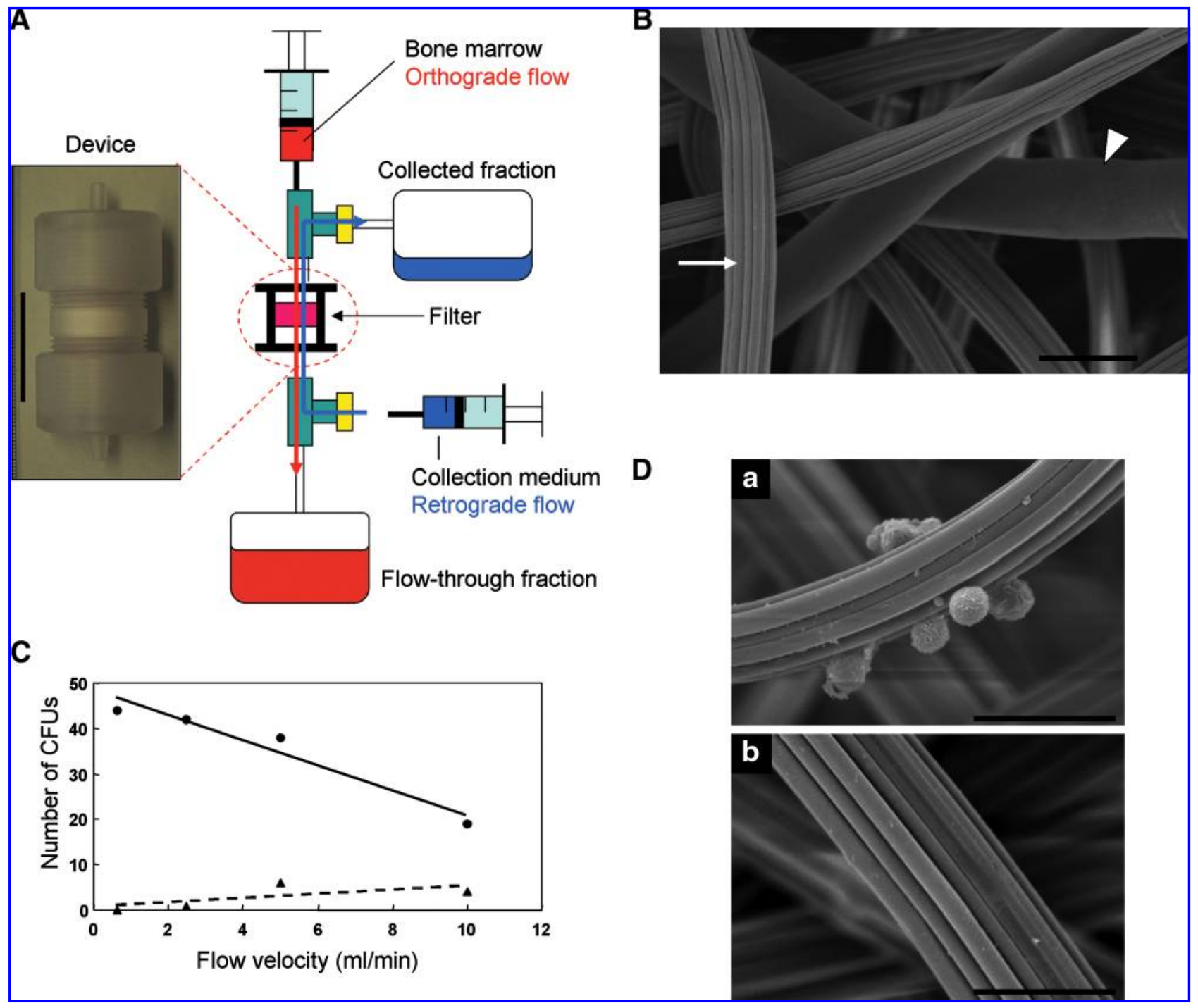

FIG. 1. Device containing a nonwoven fabric filter. (A) Photo of the device and schematic explanation of the collection method. The red line indicates the orthograde flow to apply bone marrow, and the blue line indicates the retrograde flow to collect cells. Scale bar $=30 \mathrm{~mm}$. (B) Electron micrograph of the nonwoven fabric of rayon and polyethylene. Arrow, rayon; arrowhead, polyethylene. Magnification, $2000 \times$. Scale bar $=20 \mu \mathrm{m}$. (C) Conditioning of flow velocity using swine bone marrow. Closed circles and closed triangles indicate the number of colony-forming units (CFUs) formed by cells in the collected fraction and flow-through fraction at the indicated flow velocity, respectively. (D) Electron micrograph of the surface of the filter after the orthograde flow of swine bone marrow (a) and after the retrograde flow of collection medium (b). Magnification, 2500×. Scale bar $=20 \mu \mathrm{m}$.

performed using the Super Script First Strand Synthesis System (Invitrogen). Polymerase chain reaction amplification was carried out using rTaq polymerase (Toyobo, Osaka, Japan). All polymerase chain reactions were performed using GeneAmp 9700 (PE Applied Biosystem, Foster City, CA) and specific primers for each gene. ${ }^{14}$

\section{Preparation of the canine model}

\section{of osteonecrosis and cell transplantation}

A canine model of scapho-lunate osteonecrosis was prepared as described previously. ${ }^{15}$ Briefly, scapho-lunates on one side were exposed dorsally, and a cortical window of $5 \times 10 \mathrm{~mm}$ was made, through which as much cancerous bone as possible was removed. After the curettage, the cavity was filled with liquid nitrogen for $10 \mathrm{~min}$, and the frozen bone was thawed at room temperature for $10 \mathrm{~min}$. This freezethaw procedure was repeated three times. On one side, the bone cavity was filled with beta-tricalcium phosphate ( $\beta$ TCP) granules $\left(200 \mu \mathrm{g}, 0.2 \mathrm{~cm}^{3}\right)\left(\right.$ Osferion $^{\circledR}$; Olympus, Tokyo, Japan) (hereafter designated the control side). On the other side, canine BMSCs $\left(6.0 \times 10^{7} \pm 1.5 \times 10^{7}\right)$ isolated by the device from $10 \mathrm{~mL}$ of bone marrow were mixed with the same amount of $\beta$-TCP and transplanted into the bone cavity (hereafter designated the BMSC side). Finally, the bone window was plugged with $5 \times 10 \mathrm{~mm}$ cortical bone. Animals were sacrificed and evaluated at 4 weeks after the operation $(n=6)$. 


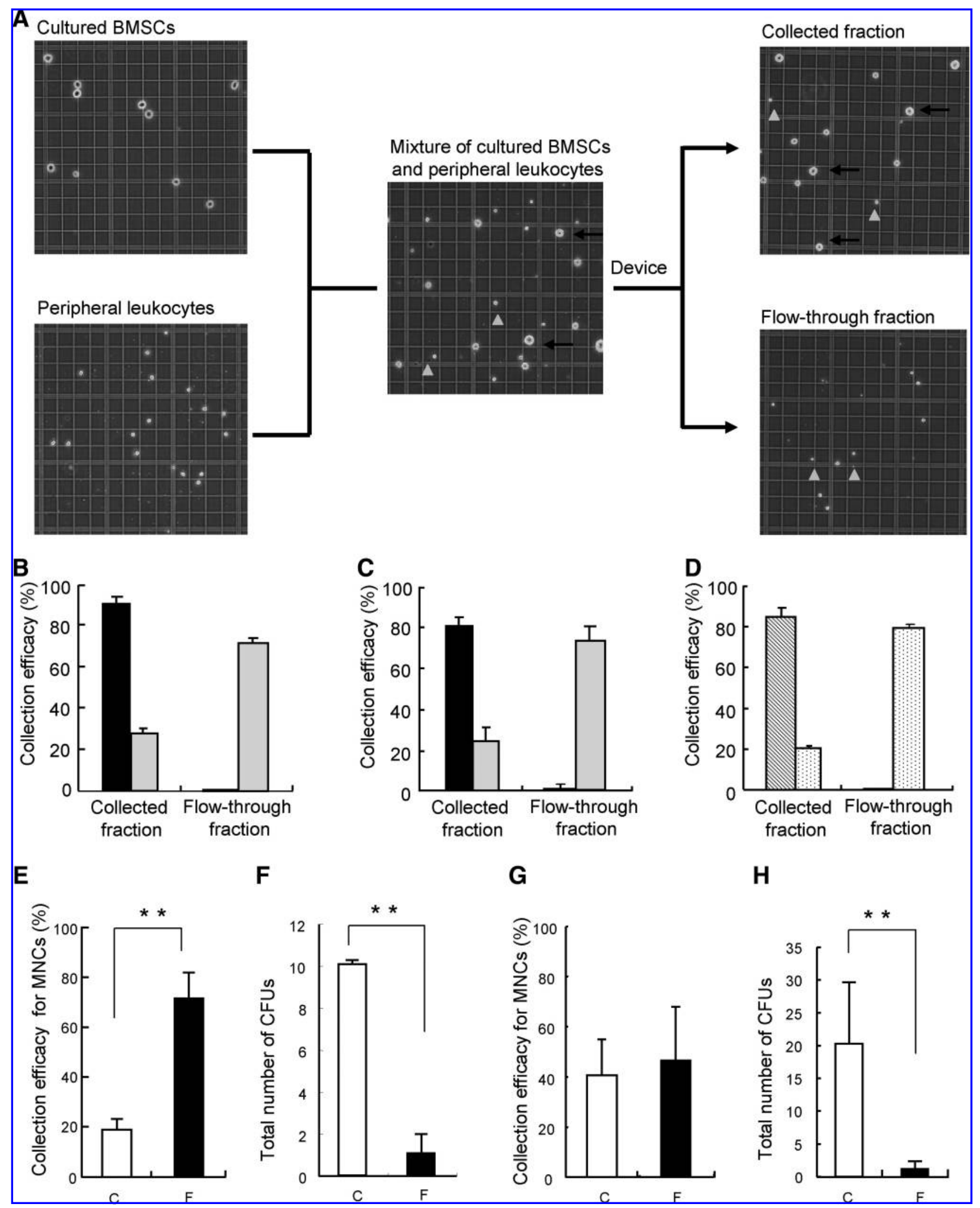




\section{Imaging analyses}

X-ray. Antero-posterior and lateral views of both wrists were obtained. The carpal height ratio was calculated from the antero-posterior view. ${ }^{15,16}$ The Ståhl index was calculated from the lateral view. ${ }^{15,17}$

Micro-computed tomography. Canine carpal bones were scanned by computed tomography (CT) (SMX-100CT-SV3 type; Shimazu, Kyoto, Japan). The image consisted of 800 slices with a voxel size of $68.224 \mathrm{~mm}$ in all three axes. Coronal and sagittal cross-sectional views of the scapho-lunate were reconstructed using adjunctive software. The same setting was used for all samples. The image reconstruction and quantification of the scapho-lunate were performed with VG Studio MAX software (Nihon Visual Science, Tokyo, Japan). ${ }^{15}$

\section{Macroscopic and microscopic assessments}

The volume and weight of extracted scapho-lunate bones were actually measured using a scale. The samples were fixed in a $10 \%$ formalin solution, decalcified with PlankRychlo solution, and then embedded in paraffin. Serial sections were cut at $4 \mu \mathrm{m}$, and stained with hematoxylin-eosin and silver impregnation.

Using a light microscope, the absorption of $\beta$-TCP granules and the formation of bone were graded as follows. ${ }^{18}$ For hematoxylin-eosin staining, granules were not surrounded by cells or newly forming bone (grade 1); granules were surrounded by osteoblasts and/or osteoclasts (grade 2); and granules were surrounded by newly forming bone (grade 3 ). For silver impregnation staining, granules were not completely surrounded by collagen fibrils (grade 1); granules were completely surrounded by collagen fibrils (grade 2); and granules were completely eroded with reticulated collagen fibrils (grade 3 ). All the $\beta$-TCP granules in a whole section were classified according to the three grades. The rates of each grade were then calculated.

\section{Statistics}

Results are expressed as the mean \pm SE. The statistical analysis was first performed by the analysis of variance (ANOVA), and if there was a significant difference among samples, Turkey's post hoc test was performed for multiple comparisons. The Student's $t$-test was carried out to compare individual data. A significant difference was accepted at $p<0.05$.

\section{Results}

\section{Screening of materials}

The first screening, of 200 potential biomaterials for the filter, was based on the parameters described in the Materials and Methods section. Forty materials were then selected for the second screening using swine bone marrow cells $(n=2)$. Ten biomaterials showed CFU counts of more than 10, and were selected for the third screening by CFU assay $(n=3)$, of which six preceded to the fourth and last screening $(n=3)$. Finally, a rayon-polyethylene nonwoven fabric was selected as the material for the filter (Supplemental Fig. S1, available online at www.liebertonline.com). First, the optimum velocity of filtration was determined by comparing CFU counts of cells in the collected and flow-through fractions. A velocity of $2.5 \mathrm{~mL} / \mathrm{min}$ was chosen for further experiments (Fig. 1C). Electron microscopic analysis confirmed the binding of swine bone marrow cells and their detachment under retrograde flow (Fig. 1D, $a$ and $b$ ).

\section{Selective collection of BMSCs by the device}

To analyze the device's selectivity, cultured human BMSCs or peripheral leukocytes were applied to it, and the cells in the collected fraction and flow-through fractions were enumerated (Fig. 2B). More than $90 \%$ of BMSCs were collected in the collected fraction, compared to only $28 \%$ of peripheral leukocytes (Fig. 2B). This selectivity was also observed when equal numbers $\left(1 \times 10^{6}\right)$ of BMSCs and peripheral leukocytes were mixed and then applied to the device (Fig. 2C). Because BMSCs and peripheral leukocytes differ in size, we could distinguish between them in the cell counting chamber (Fig. 2A). Approximately $80 \%$ of BMSCs were collected in the collected fraction, and an almost equal percentage of peripheral leukocytes were collected in the flowthrough fraction (Fig. 2C). More than $90 \%$ of $\mathrm{CD}_{4} 4^{+}$cells were collected with the device, leaving almost no $\mathrm{CD} 44^{+}$ cells in the flow-through fraction, whereas less than $20 \%$ of $\mathrm{CD}_{4} 5^{+}$cells were collected (Fig. 2D). These results suggested the selective collection of BMSCs by the device.

FIG. 2. Selective collection of bone marrow stromal cells (BMSCs) with CFUs by the device. (A) Selective counting of BMSCs and peripheral leukocytes. BMSCs and peripheral leukocytes differed in morphology in the cell counting chamber (left two photos). After the mixing of the two types of cells (middle) and application of the device, each type of cell in the collected (right upper) or flow-through (right lower) fraction was numerated. Arrow, cultured BMSCs; arrowhead, peripheral leukocytes. (B) Collection efficacy for human BMSCs (black box) and peripheral leukocytes (gray box). Each type of cell was applied to the device separately, and collection efficacy was calculated from the number of cells in the collected and flowthrough fractions. (C) Collection efficacy for human BMSCs (black box) and peripheral leukocytes (gray box). Equal numbers of BMSCs and peripheral leukocytes were mixed and applied to the device. Collection efficacy was calculated from the number of cells in the collected and flow-through fractions. (D) Collection efficacy for CD44 (hatched box) and CD45 ${ }^{+}$(dotted box) cells. Equal numbers of BMSCs and peripheral leukocytes were mixed and applied to the device. Cells positive for CD44 or CD45 in the collected or flow-through fraction were numerated by fluorescence-activated cell sorting (FACS), and collection efficacy was calculated from the number of positive cells in the cell mixture before the treatment. (E) Collection efficacy for mononuclear cells (MNCs) from canine bone marrow. ${ }^{* *} p<0.01$ (F) Total number of CFUs derived from canine MNCs collected in the collected and flow-through fractions. From the results on collection efficacy and number of CFUs, the total number of CFUs was calculated. ${ }^{* *} p<0.01$. (G) Collection efficacy for MNCs from human bone marrow. (H) Total number of CFUs derived from human MNCs collected in the collected and flow-through fractions. ${ }^{* *} p<0.01$. 


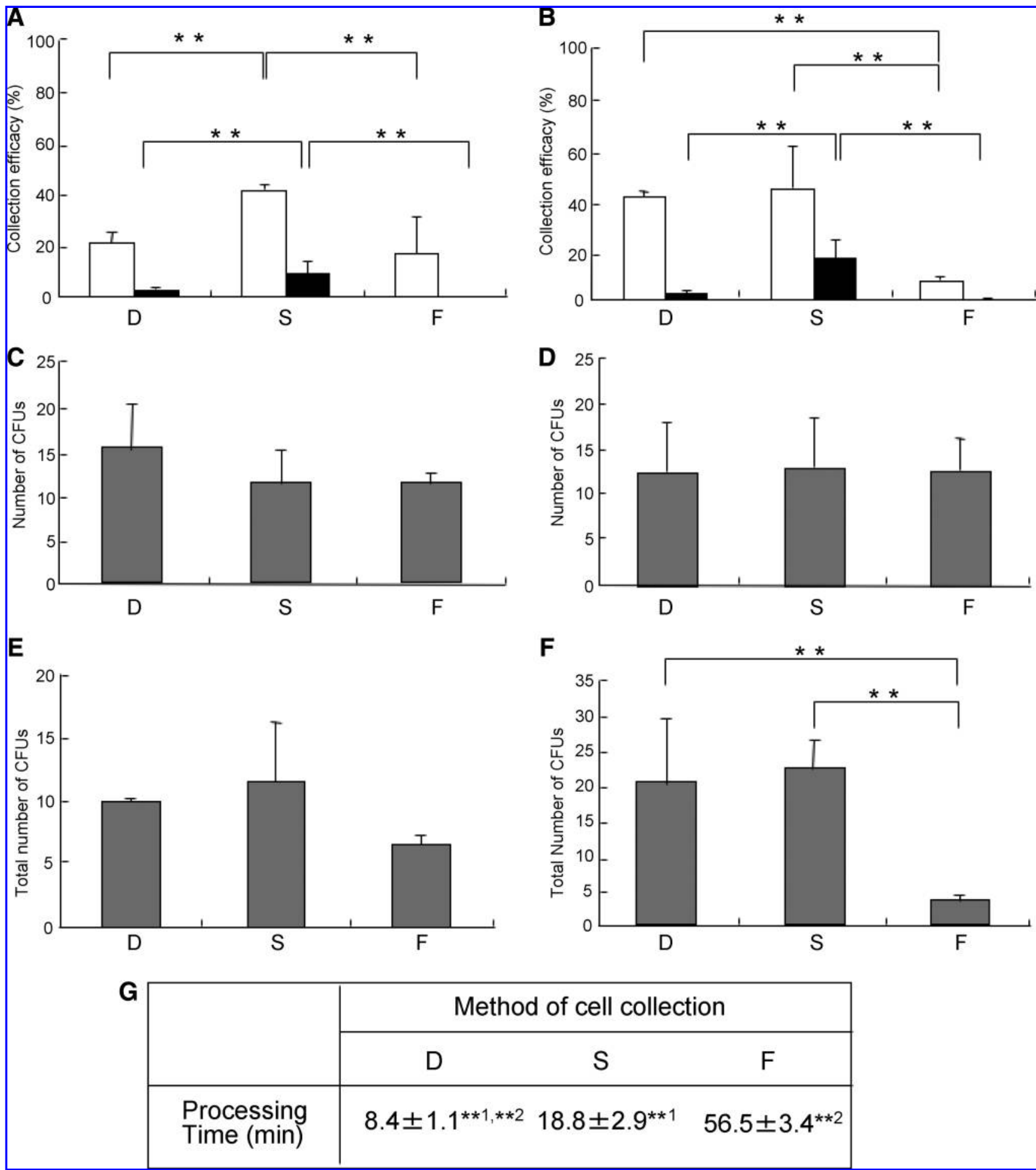

FIG. 3. Device selectively prepares MNCs with CFUs. Collection efficacy for MNCs (white box) and erythrocytes (black box) isolated with each method from canine (A) and human (B) bone marrow. D, S, or F indicates each method described in Materials and Methods section. ${ }^{* *} p<0.01$. Number of CFUs derived from equal numbers $\left(2.5 \times 10^{5}\right)$ of MNCs isolated by each method from canine (C) and human (D) bone marrow. Total number of CFUs derived from equal amounts of canine (E) and human $(\mathbf{F})$ bone marrow using each method. ${ }^{* *} p<0.01$. (G) Processing time for each method. ${ }^{* * 1} p<0.01 ;{ }^{* * 2} p<0.01$.

\section{Selective collection of colony-forming cells from bone marrow by the device}

Next, the selectivity of the device was analyzed using cells derived from canine (Fig. 2E, F) and human (Fig. 2G, H) bone marrow. In both cases, most erythrocytes passed through the filter and were collected in the flow-through fraction. Only a few were found in the collected fraction (data not shown). For canine bone marrow, the number of MNCs was significantly smaller in the collected fraction than in the 
flow-through fraction $(p=0.002$; Fig. 2E). The number of CFUs, however, was much higher in the collected fraction $(p<0.001$; Fig. 2F). For human bone marrow, the number of MNCs in the collected and flow-through fraction did not differ (Fig. 2G). However, the number of CFUs was much higher in the collected fraction than in the flow-through fraction $(p<0.001$; Fig. $2 \mathrm{H})$. These results indicated the selective collection of CFUs by the device.

\section{Comparison of method $D$ with methods $S$ and $F$}

The biological properties of cells isolated by each of the three methods were compared. First, we compared the collection efficacy of method D with that of method S or F. ANOVA revealed significant differences among the collection efficacies for MNCs (canine, $p=0.024$; human, $p=0.0002$ ). Comparisons between individual methods were then carried out using Turkey's post hoc test. For canine bone marrow, the collection efficacy for MNCs was significantly lower with method D $(20.7 \pm 4.2 \%)$ than method S $(41.6 \pm 1.6 \%)(p<0.01$; Fig. 3A), and the same between method $\mathrm{D}$ and method $\mathrm{F}(16.8 \pm 14.0 \%)$ (Fig. 3A). As for contamination by erythrocytes, method S showed larger numbers than method D or F. For human bone marrow, there was no difference in collection efficacy between method D $(40.5 \pm 14.5 \%)$ and method $S(43.9 \pm 10.8 \%)$ (Fig. $3 B)$, and method $\mathrm{F}(7.3 \pm 1.8 \%)$ was significantly less efficient than method D $(p<0.01$; Fig. 3B). The number of contaminating RBCs was again high with method S (Fig. 3B). Next, the MNCs collected by each method were seeded on plastic dishes, and the number of CFUs was counted 14 days later. ANOVA indicated no significant difference among the numbers of CFUs of MNCs prepared by the three methods in canine $(p=0.40)$ and human bone marrow $(p=0.95)$ (Fig. 3C, D). From the results on collection efficacy and number of CFUs, the total number of CFUs isolated from bone marrow was calculated for each method. In the case of canine bone marrow, the total number of CFUs with method $\mathrm{D}(10.1 \pm 0.2)$ was equivalent to that with method $S(11.7 \pm 4.7)$ and much larger than that with method F, but not significant (6.6 \pm 0.1$)$ (Fig. 3E). Similar results were obtained with human bone marrow cells. The total number of CFUs with method D $(20.3 \pm 9.4)$ was equivalent to that with method $S(22.3 \pm 4.4)$, and much larger than that with method F $(3.6 \pm 1.0)(p<0.01 ;$ Fig. 3F). These results indicated that the device collected colony-forming cells from bone marrow with an efficacy equal to that of current methods requiring centrifugation. In addition, the processing time for method D $(8.4 \pm 1.1 \mathrm{~min}$; Fig. $3 \mathrm{G})$ was significantly shorter than that for the other methods (method $S, 18.8 \pm 2.9 \mathrm{~min}$; method F, $56.5 \pm 3.4 \mathrm{~min}$ ) (ANOVA, $p<0.001$; Turkey's post hoc test, $p<0.01$; Fig. 3G).

\section{Expression of MSC markers in isolated BMSCs}

To compare the cell population isolated by each of the three methods, the expression of cell surface markers was analyzed by fluorescence-activated cell sorting (FACS). Because a combination of markers is more reliable, we focused on $\mathrm{CD} 10^{+} / \mathrm{CD} 271^{+}$, $\mathrm{CD}^{+} 0^{+} / \mathrm{CD} 271^{+}$, or $\mathrm{CD} 106^{+} / \mathrm{STRO}-1^{+}$double-positive cells. The $\mathrm{CD} 0^{+} / \mathrm{CD} 271^{+}$and the $\mathrm{CD} 106^{+} / \mathrm{STRO}-1^{+}$populations were significantly larger among cells collected by method $\mathrm{D}$ than those collected by method S or method F (ANOVA, $p<0.001$; Turkey's post hoc test, $p<0.01$ for $\mathrm{CD}^{+} 0^{+} / \mathrm{CD} 271^{+}, p<0.05$ for $\mathrm{CD} 106^{+} / \mathrm{STRO}^{-}{ }^{+}$, respectively; Fig. $\left.4 \mathrm{~A}, \mathrm{~B}\right)$.

\section{Differentiation potential of isolated BMSCs}

To analyze their potential to differentiate, the BMSCs isolated by each of the methods were propagated on culture

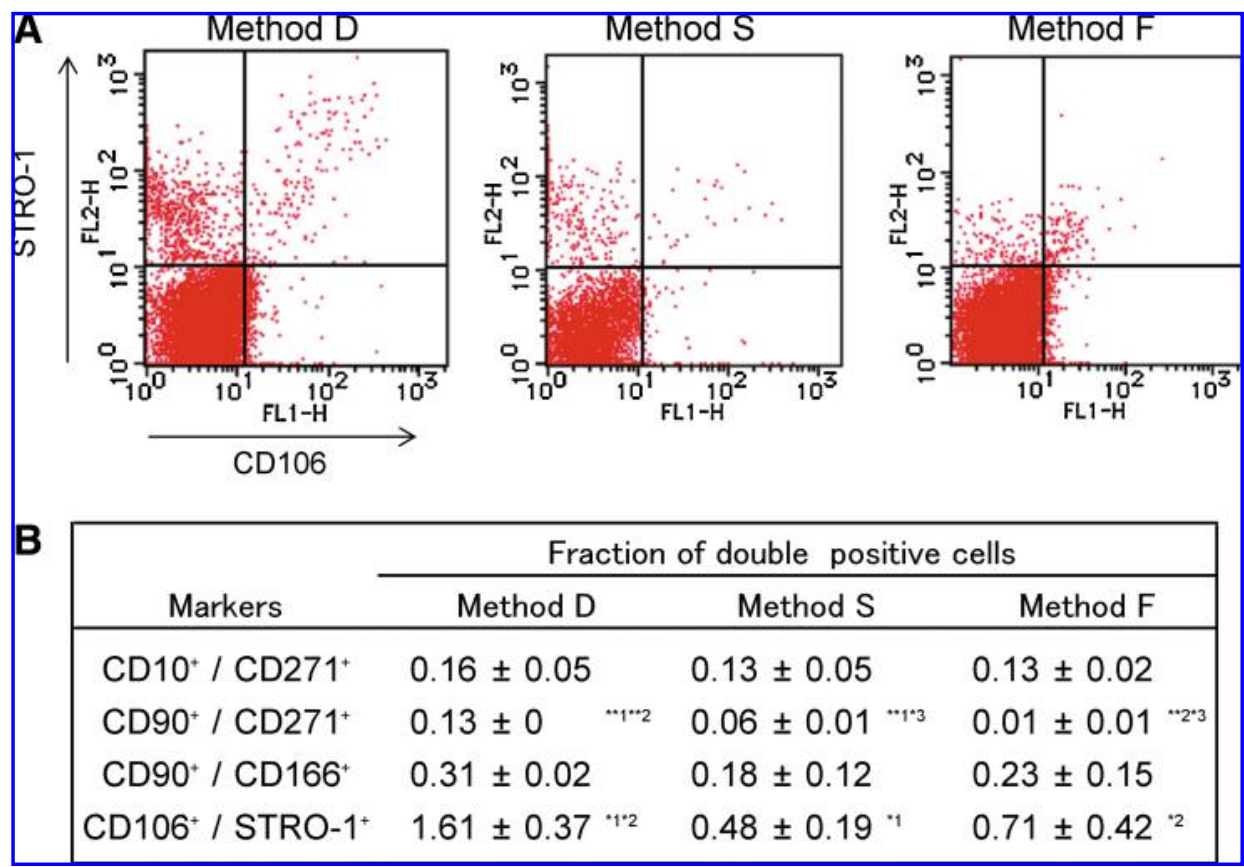

FIG. 4. Device selectively prepares MNCs, which express mesenchymal marker. (A) FACS analysis of CD106 and STRO-1 on MNCs isolated by each method. (B) Fraction of cells double-positive for mesenchymal stem cell (MSC) markers among MNCs isolated by each method. ${ }^{* *} 1,{ }^{* *} 2 p<0.01 ;{ }^{*} 1,2,{ }^{*} 3 p<0.05$. 

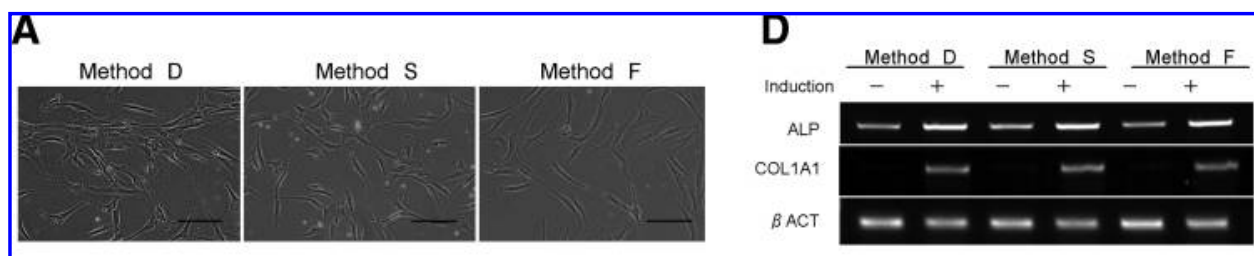

$\mathbf{F}$
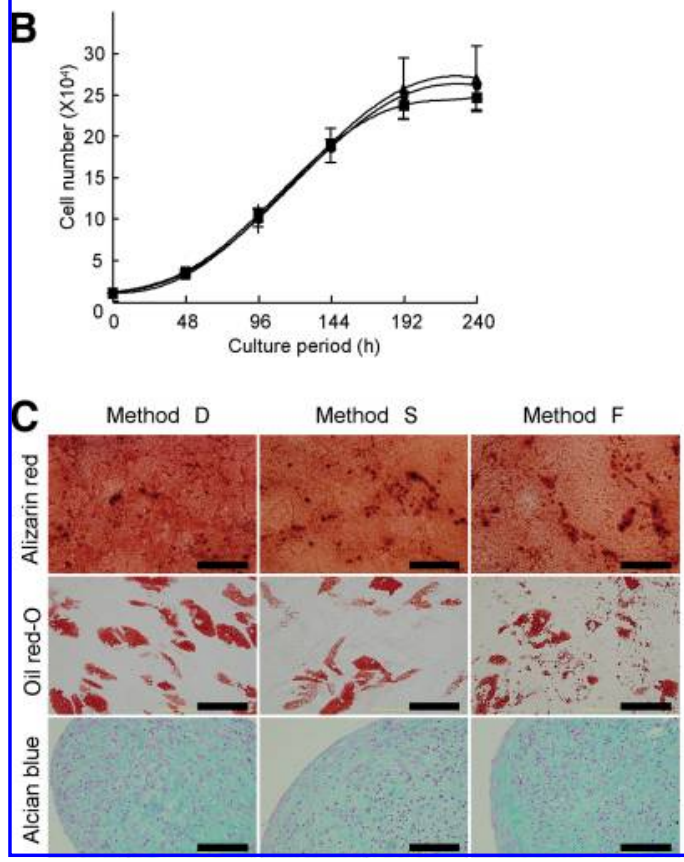

H

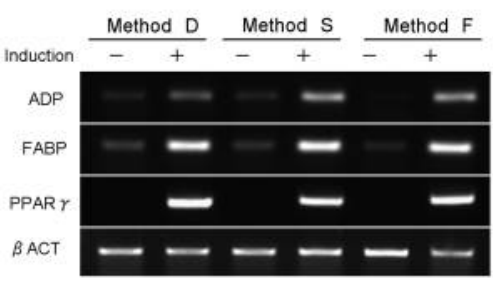

G
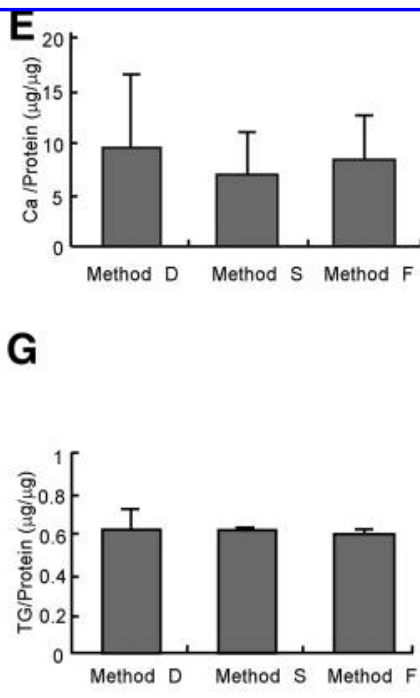

I
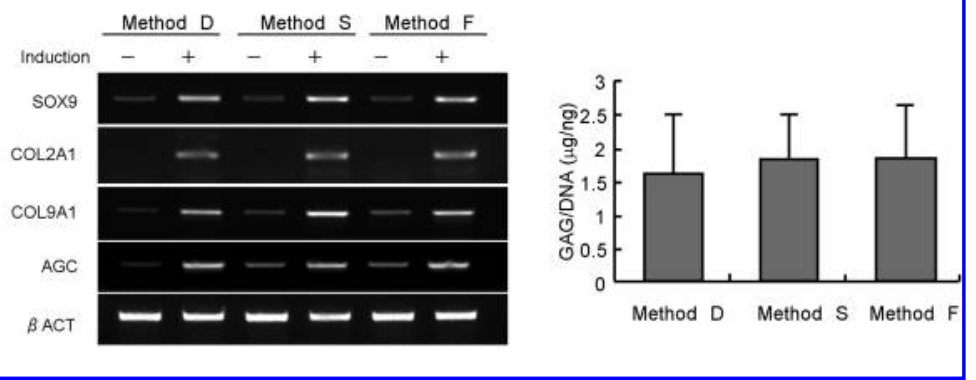

FIG. 5. Growth and differentiation potential of human BMSCs isolated by each method. The same numbers of BMSCs isolated by the three methods from human bone marrow were propagated on culture dishes for 2 weeks under standard conditions. (A) Microscopic views of cultured BMSCs isolated by each method. Magnification, $100 \times$. Scale bars $=100 \mu \mathrm{m}$. $(\mathbf{B})$ Growth of cultured BMSCs isolated by each method. $\bullet$, Method D; $\boldsymbol{\Delta}$, method S; $\boldsymbol{\square}$, method F. (C) Differentiation potential of BMSCs isolated by each method. Osteogenic differentiation was analyzed by alizarin red staining. Magnification, $100 \times$. Scale bars $=100 \mu \mathrm{m}$. Adipogenic differentiation was analyzed by oil red-O staining. Magnification, $100 \times$. Scale bars $=100 \mu \mathrm{m}$. Chondrogenic differentiation was analyzed by alcian blue staining. Magnification, $40 \times$. Scale bars $=300 \mu \mathrm{m}$. (D) Expression of bone-related genes before and after osteogenic induction. $A L P$, alkaline phosphatase. (E) Ca content after osteogenic induction. Ca, calcium. (F) Expression of fat-related genes before and after adipogenic induction. $A D P$, adipsin; $F A B P$, fatty acid-binding protein; $P P A R \gamma$, peroxisome proliferator-activated receptor gamma. (G) TG content after adipogenic induction. TG, triglyceride. (H) Expression of cartilage-related genes before and after chondrogenic induction. AGC, aggrecan. (I) GAG content after chondrogenic induction. GAG, glycosaminoglycan.

dishes and incubated for 2 weeks under standard growth conditions for MSCs. They were then induced to undergo osteogenesis, adipogenesis, and chondrogenesis. The morphology of the cells isolated by each of the methods was almost the same for both canine (Supplemental Fig. S2A, available online at www.liebertonline.com) and human (Fig. 5A) BMSCs. There was no difference in growth profile among the three methods in canine (Supplemental Fig. S2B, available online at www.liebertonline.com) or human (Fig. 5B) BMSCs. Osteogenic differentiation potential was evaluated with histochemical (alizarin red staining; Fig. 5C), mRNA expression (expression of the ALP and COL1A1 genes; Fig. 5D), and biochemical (calcium content; Fig. 5E) analyses. There was no significant difference among the cells isolated by each of the three methods (ANOVA, $p=0.61$ ). Similar results were observed in adipogenic differentiation

FIG. 6. Regeneration of bone tissues by cells isolated with the device. Data for six dogs at 4 weeks after the operation are presented. Frontal (A) and sagittal (B) views of micro-computed tomography. (C) Morphometrical analyses. The results of Xray and micro-computed tomography were evaluated using the criteria described in the Materials and Methods section. Bone weight and volume were actually measured. ${ }^{* *} p<0.01$; ${ }^{*} p<0.05$. CHR, carpal height ratio. (D) Histological analyses by hematoxylin-eosin staining. Magnification, $100 \times$. Scale bar $=500 \mu \mathrm{m}$. (E) Fraction of regenerated bone tissue of each grade. Grade 1, white box; grade 2, gray box; grade 3, black box. ${ }^{*} p<0.05$. (F) Histological analyses using silver impregnation staining. Magnification, $200 \times$. Scale bar $=200 \mu \mathrm{m}$. (G) Fraction of $\beta$-tricalcium phosphate with integrated collagen fibers of each grade. Grade 1, white box; grade 2, gray box; grade 3, black box. ${ }^{*} p<0.05$. 


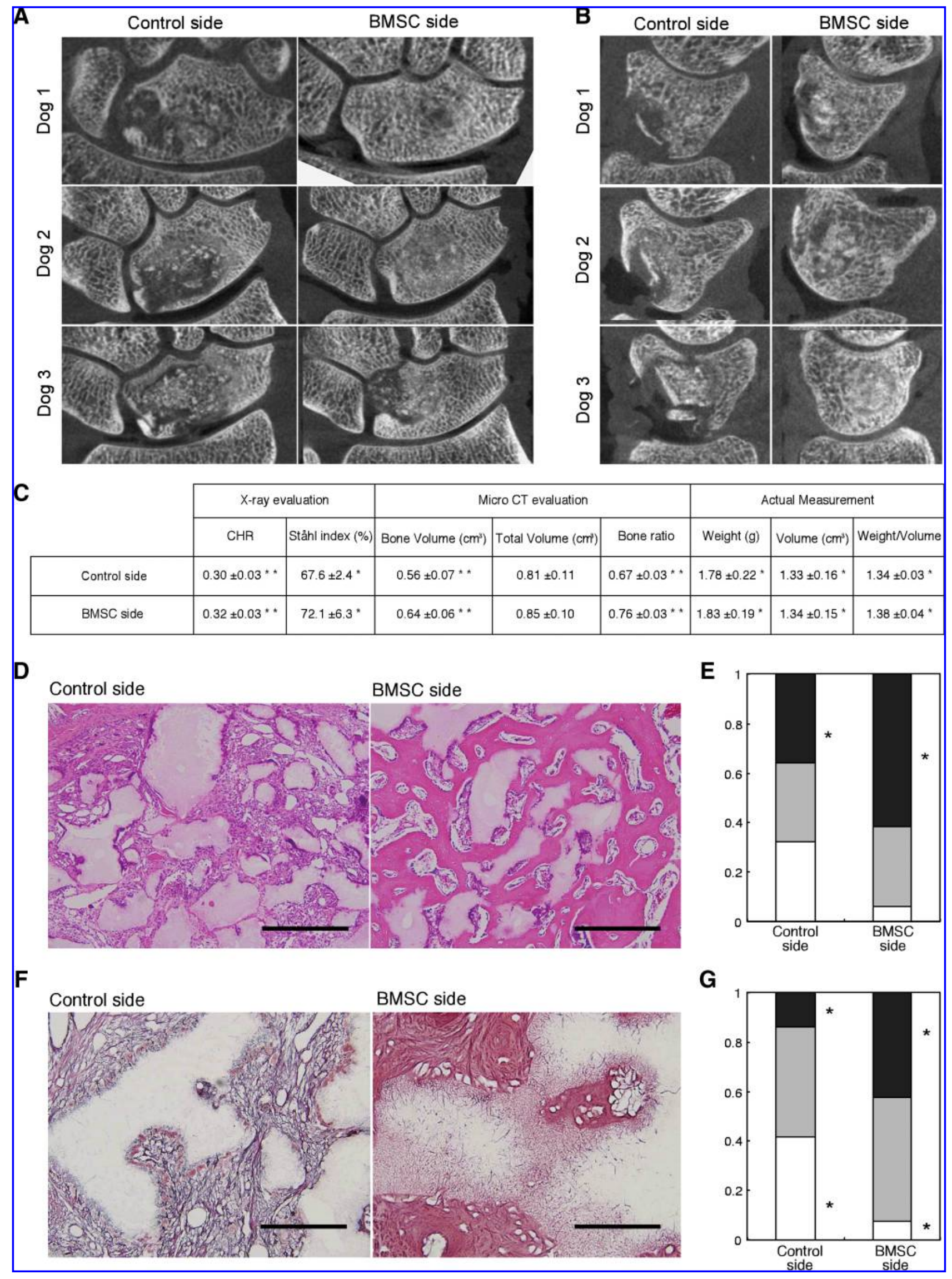


(ANOVA, $p=0.41$; Fig. 5C, F, G) and chondrogenic differentiation (ANOVA, $p=0.97$; Fig. 5C, H, I). Same results were obtained in the analyses of canine BMSCs (Supplemental Fig. S2C, available online at www.liebertonline.com). These results suggested that BMSCs isolated by each of the three methods had the multidirectional differentiation potential of MSCs.

\section{Regeneration of bone tissues by cells isolated with the device}

To confirm the in vivo osteogenic potential of cells collected with the device, we used the cells to regenerate bone tissues in a model of osteonecrosis using canine scapho-lunates, which we established previously. ${ }^{15}$ Micro-CT findings at 4 weeks after the operation indicated bone regeneration to be more prominent on the BMSC side than control side in all cases (Fig. 6A, B). Carpal height ratio and the Ståhl index calculated from plain X-rays show the degree of collapse of scapholunate bone. Both parameters were significantly higher on the BMSC side than control side (Fig. 6C). Total volume and bone volume were analyzed from the micro-CT data. Bone volume and therefore bone ratio were greater on the BMSC side (Fig. $6 \mathrm{C})$. Both actual weight and volume were also significantly higher on the BMSC side (Fig. 6C). These quantitative analyses suggested that the application of BMSCs prepared by the device contributed significantly to bone regeneration.

After the physical and radiological examinations, samples were processed for histological analyses. Microscopic findings of samples taken from the control side showed that a large amount of $\beta$-TCP remained unabsorbed, although multinucleated giant cells were found adjacent to $\beta$-TCP. In contrast, abundant new bone with the active absorption of $\beta$ TCP by multinucleated giant cells was found on the BMSC side (Fig. 6D). The extent to which $\beta$-TCP was absorbed as new bone formed was scored using criteria described in the Materials and Methods section. The grade 3 fraction was significantly larger on the BMSC side ( $p=0.032$; Fig. 6E).

To analyze the interaction between the prepared cells and $\beta$-TCP, silver impregnation staining was performed (Fig. $6 \mathrm{~F}$ ). The staining shows the invasion of collagen fibers into biodegradable materials. Collagen fibers in $\beta$-TCP granules adjacent to regenerated bone tissue were abundant on the BMSC side, but rare on the control side (Fig. 6F). Quantitative analyses showed the extent of the invasion to be significantly greater on the BMSC side ( $p=0.034$; Fig. 6G). These results suggested that BMSCs prepared using the device enhanced bone regeneration in vivo.

\section{Discussion}

Bone marrow cells consist of two types of MNCs: hematopoietic cells including hematopoietic stem cells, and stromal cells including MSCs. Approximately 99\% of MNCs in bone marrow belong to the former population, and among the stromal cells, less than $5 \%$ have multidirectional differentiation potential compatible with the concept of MSCs. Therefore, the proportion of MSCs among bone marrow MNCs is estimated at less than $0.05 \% .{ }^{19}$ To obtain BMSCs including MSCs from bone marrow, it is essential to separate the hematopoietic MNCs.

Takenaka developed a novel filter composed of nonwoven fabrics to trap peripheral leukocytes for the treatment of autoimmune disease. ${ }^{10}$ Because erythrocytes are deformed, which allows them to pass through small pores and have low adhesiveness, they can be separated from MNCs by the filter. ${ }^{10}$ They used fibers $1.7 \mu \mathrm{m}$ in diameter, because all the leukocytes were trapped when the fiber was less than $3 \mu \mathrm{m}$ in diameter. ${ }^{10}$ This filter, however, is not applicable to the separation of MSCs from hematopoietic MNCs, both of which will be captured, making it difficult to separate them by cell size. Therefore, we used fibers $15 \mu \mathrm{m}$ in diameter with greater affinity for BMSCs than hematopoietic MNCs. MSCs are more adherent than hematopoietic cells. ${ }^{4,19}$ MSCs adhere much more tightly to highly hydrophilic and rough surfaces than to hydrophobic and smooth surfaces. ${ }^{20}$ The contact angle of the nonwoven fabric in the current device is estimated to be $20^{\circ}$, which is highly hydrophilic and may make it possible to trap MSCs in the device. This adherent trapping method may prevent excess shearing stress caused by the flow. Cells isolated by the device showed no increase in bradykinine or lactate dehydrogenase (LDH) (data not shown). In addition, mRNA expression of the $p 16$ and $p 53$ genes was not increased, and the karyotype showed no abnormalities (data not shown).

In terms of its potential clinical applications, the device has several advantages. The filter is composed of rayon and polyethylene. Rayon is used for the dialysis membranes of hemodialyzers and known to decrease platelet activity. ${ }^{21}$ Polyethylene is a biomaterial employed in total hip replacement and as a scaffold for tissue engineering. ${ }^{22}$ Therefore, all the materials are safe for clinical use. The isolation of BMSCs in a closed system is a major advantage of this device. Centrifugation requires mixing with an appropriate solution, which has to be performed in an open system. Current guidelines require the centrifugation process to be performed in an isolated area with specialized equipment. Because all steps can be done in a closed system, the device can isolate BMSCs from bone marrow on the operation table. Further, processing time is shorter than for the other two methods (Fig. 3G), which may help to reduce cell death during the isolation procedures. In addition, the method does not require special skills, which will help to obtain constant results and also be an advantage for clinical application.

We have shown that in terms of differentiation potential, the cells isolated using the device were equivalent to those prepared by current methods using centrifugation. The total number of CFUs obtained from bone marrow was equal to that achieved with method $S$ and higher than that obtained with method F, suggesting that the device recovers almost all cells with the ability to form CFUs. FACS analyses showed that the method was superior to others in terms of the recovery of the MSC marker-positive population. CD10, CD44, CD90, CD106, and STRO-1 are considered surface markers of MSCs, ${ }^{13,23}$ and, recently, CD271 was identified as a marker of MSCs. ${ }^{24}$ The fractions of $\mathrm{CD} 90^{+} / \mathrm{CD} 271^{+}$cells were larger among cells collected using the device than those isolated with the other two methods (Fig. 4). The CD106 ${ }^{+} /$STRO- $1^{+}$ fraction $(1.61 \pm 0.37 \%$; Fig. 4$)$ was even larger than that collected by FACS $(1.4 \pm 0.3 \%){ }^{25}$ which has a risk of microbial contamination and unfavorable effects. We have no clear explanation for this selective collection. Because CD106 ${ }^{+} / \mathrm{STRO}-1^{+}$ cells attach to plastic dish efficiently and have high colonyforming activity, ${ }^{26}$ one possibility is that $\mathrm{CD} 106^{+} / \mathrm{STRO}-1^{+}$cells have high affinity to the filter materials used in the device. Finally, we have shown that the direct application of the trapped cells without ex vivo cultivation assisted the regeneration of bone 
tissue in vivo (Fig. 6), although the role of transplanted cells may not be simply to reconstitute bone tissues by themselves but also to produce cytokines stimulating host cells, ${ }^{15}$ suggesting that BMSCs collected with the new device on the operating table could be used for conditions requiring an acceleration of bone regeneration such as the nonunion of bone fractures.

\section{Acknowledgments}

We thank Dr. H. Iwata for suggestions and Drs. M. Neo, S. Fujibayashi, M. Takemoto, H. Ito, and H. Yoshitomi for clinical samples. This work was supported by Grants-in-aid for Scientific Research from the Japan Society for the Promotion of Science; from the Ministry of Education, Culture, Sports, Science, and Technology; from the Ministry of Health, Labor, and Welfare; and from the New Energy and Industrial Technology Development Organization.

\section{Disclosure Statement}

No competing financial interests exist.

\section{References}

1. Caplan, A.I. Mesenchymal stem cells. J Orthop Res 9, 641, 1991.

2. Wagner, W., and Ho, A.D. Mesenchymal stem cell preparations comparing apples and oranges. Stem Cell Rev 3, 239, 2007.

3. Peterson, E.A., and Evans, W.H. Separation of bone marrow cells by sedimentation at unit gravity. Nature 214, 824, 1967.

4. Caterson, E.J., Nesti, L.J., Danielson, K.G., and Tuan, R.S. Human marrow-derived mesenchymal progenitor cells: isolation, culture expansion, and analysis of differentiation. Mol Biotechnol 20, 245, 2002.

5. Li, Y., Ma, T., Yang, S.T., and Kniss, D.A. Thermal compression and characterization of three-dimensional nonwoven PET matrices as tissue engineering scaffolds. Biomaterials 22, 609, 2001.

6. Takahashi, Y., and Tabata, Y. Effect of the fiber diameter and porosity of non-woven PET fabrics on the osteogenic differentiation of mesenchymal stem cells. J Biomater Sci Polym Ed 15, 41, 2004

7. $\overline{M a}$, Z., Kotaki, M., Yong, T., He, W., and Ramakrishna, S. Surface engineering of electrospun polyethylene terephthalate (PET) nanofibers towards development of a new material for blood vessel engineering. Biomaterials 26, 2527, 2005.

8. Gador, W., and Jankowska, E. Filtration properties of nonwovens. Int J Occup Saf Ergon 5, 361, 1999.

9. Gorman, N.E. Nonwoven materiel: manufacturing processes for sterilization wraps. Hosp Mater Manage Q 9, 1, 1988.

10. Takenaka, Y. Lymphocytapheresis. Artif Organs 20, 914, 1996.

11. Onodera, H., Abe, Y., Yoshida, M., Yamawaki, N., Yamashita, Y., Matsuo, H., Ichinose, K., Otsuru, I., and Shibuya, $\mathrm{N}$. A new device for selective removal of $\mathrm{CD} 4^{+} \mathrm{T}$ cells. Ther Apher 2, 37, 1998.

12. Shibata, K.R., Aoyama, T., Shima, Y., Kenichi, F., Otsuka, S., Furu, M., Kohne, Y., Ito, K., Fujibayashi, S., Meo, M., Nakayama, T., Nakamura, T., and Toguchida, J. Expression of the p16INK4A gene is associated closely with senescence of human mesenchymal stem cells and is potentially silenced by DNA methylation during in vitro expansion. Stem Cells 25, 2371, 2007.

13. Pittenger, M.F., Mackay, A.M., Beck, S.C., Jaiswal, R.K., Douglas, R., Mosca, J.D., Moorman, M.A., Simonetti, D.W., Craig, S., and Marshak, D.R. Multilineage potential of adult human mesenchymal stem cells. Science 284, 143, 1999.
14. Fukiage, K., Aoyama, T., Shibata, K.R., Otsuka, S., Furu, M., Kohno, Y., Ito, K., Jin, Y., Fujita, S., Fujibayashi, S., Neo, M., Nakayama, T., Nakamura, T., and Toguchida, J. Expression of vascular cell adhesion molecule- 1 indicates the differentiation potential of human bone marrow stromal cells. Biochem Biophys Res Commun 365, 406, 2008.

15. Ikeguchi, R., Kakinoki, R., Aoyama, T., Shibata, K.R., Otsuka, S., Fukiage, K., Nishijo, K., Ishibe, T., Shima, Y., Otsuki, B., Azuma, T., Tsutsumi, S., Nakayama, T., Otsuka, T., Nakamura, T., and Toguchida, J. Regeneration of osteonecrosis of canine scapholunate using bone marrow stromal cells: possible therapeutic approach for Kienböck disease. Cell Transplant 15, 411, 2006.

16. Youm, Y., McMurthy, R.Y., Flatt, A.E., and Gillespie, T.E. Kinematics of the wrist. I. An experimental study of radial-ulnar deviation and flexion-extension. J Bone Joint Surg Am 60, 423, 1978.

17. Goldfarb, C.A., Hsu, J., Gelberman, R.H., and Boyer, M.I. The Lichtman classification for Kienböck's disease: an assessment of reliability. J Hand Surg Am 28, 74, 2003.

18. Ogose, A., Kondo, N., Umezu, H., Hotta, T., Kawashima, H., Tokunaga, K., Ito, T., Kudo, N., Hoshino, M., Gu, W., and Endo, N. Histological assessment in grafts of highly purified beta-tricalcium phosphate (OSferion) in human bones. Biomaterials 27, 1542, 2006.

19. Chamberiain, G., Fox, J., Ashton, B., and Middleton, J. Concise review: mesenchymal stem cells: their phenotype, differentiation capacity, immunological features, and potential for homing. Stem Cells 25, 2739, 2007.

20. Kim, M.S., Shin, Y.N., Cho, M.H., Kim, S.H., Kim, S.K., Cho, Y.H., Khang, G., Lee, I.W., and Lee, H.B. Adhesion behavior of human bone marrow stromal cells on differentially wettable polymer surfaces. Tissue Eng 13, 2095, 2007.

21. Thijs, A., Grooteman, M.P., Zweegman, S., Nubé, M.J., Huijgens, P.C., and Stehouwer, C.D. Platelet activation during haemodialysis: comparison of cuprammonium rayon and polysulfone membranes. Blood Purif 25, 389, 2007.

22. Tessmar, J.K., and Göpferich, A.M. Customized PEGderived copolymers for tissue-engineering applications. Macromol Biosci 7, 23, 2007.

23. Deans, R.J., and Moseley, A.B. Mesenchymal stem cells: biology and potential clinical uses. Exp Hematol 28, 875, 2000.

24. Bühring, H.J., Battula, V.L., Treml, S., Schewe, B., Kanz, L., and Vogel, W. Novel markers for the prospective isolation of human MSC. Ann NY Acad Sci 1106, 262, 2007.

25. Gronthos, S., and Zannettino, A.C. A method to isolate and purify human bone marrow stromal stem cells. Methods Mol Biol 449, 45, 2008.

26. Gronthos, S., Zannettino, A.C., Hay, S.J., Shi, S., Graves, S.E., Kortesidis, A., and Simmons, P.J. Molecular and cellular characterisation of highly purified stromal stem cells derived from human bone marrow. J Cell Sci 116, 1827, 2003.

Address correspondence to: Tomoki Aoyama, M.D., Ph.D. Institute for Frontier Medical Sciences Kyoto University 53 Kawahara-cho Shogoin, Sakyo-ku Kyoto 606-8507 Japan

E-mail: blue@frontier.kyoto-u.ac.jp

Received: December 21, 2008 Accepted: April 13, 2009

Online Publication Date: July 6, 2009 


\section{This article has been cited by:}

1. Maria Margarida Diogo, Cláudia Lobato da Silva, Joaquim M.S. Cabral. 2012. Separation technologies for stem cell bioprocessing. Biotechnology and Bioengineering n/a-n/a. [CrossRef]

2. Barbara Dozza, Claudia Di Bella, Enrico Lucarelli, Gianluca Giavaresi, Milena Fini, Pier Luigi Tazzari, Sandro Giannini, Davide Donati. 2011. Mesenchymal stem cells and platelet lysate in fibrin or collagen scaffold promote non-cemented hip prosthesis integration. Journal of Orthopaedic Research 29:6, 961-968. [CrossRef]

3. Daisuke Kanematsu, Tomoko Shofuda, Atsuyo Yamamoto, Chiaki Ban, Takafumi Ueda, Mami Yamasaki, Yonehiro Kanemura. 2011. Isolation and cellular properties of mesenchymal cells derived from the decidua of human term placenta. Differentiation . [CrossRef]

4. Helena Motaln, Cristian Schichor, Tamara T. Lah. 2010. Human mesenchymal stem cells and their use in cell-based therapies. Cancer 116:11, 2519-2530. [CrossRef] 